\title{
MARTIN SIHLES SCHULE DER INNEREN MEDIZIN IN LETTLAND
}

Geistige Begrenztheit hemmt den Weitblick, zwingt sich in Bagatellen zu vertiefen, verhindert das Gesamtbild zu erfassen. Einen solchen Wanderer interessieren weder Landschaft noch l.and und leute, ausschliesslich nur das, was in seiner nächsten Nahe vorgeht.

Andre Gide: "Stirls und Werde"

Die Verschiedenheit ähnlicher Vorgänge und die gemeinsamen Anzeichen unterschiedlicher Prozesse sind schwieriger feststellbar, falls sie sich bei der Analyse vieler Geschehnisse in großen Ländern offenbaren, bei großen Menschengruppen und überhaupt in großen Dingen. Lettland zählt zu kleinen Ländern.

Daher sind hier verschiedene Strömungen, verschiedene Menschen und Denkarten leicht zu merken. In der Wissenschaftskultur und der Gesamtkultur Lettlands gehört Jänis Stradinš zu jenen Persönlichkeiten, deren Ansichten, oft genug auch Worte und Taten, alle Gebiete umfassen, auch die Geschichte der Medizin, der seinerzeit Prof. Pauls Stradinš die Basis gelegt hatte.

Gemeinsame Ansichten und Freundschaft verbanden den Chirurgen Pauls Stradin,̌̌ und den Internisten und Lexikographen der Medizin Prof. Kristaps Rudzitis, dessen Beitrag zur Medizin und Kultur Lettlands gleichfalls beachtenswert ist.

In dieser Abhandlung beabsichtige ich, mich demjenigen Platz zuzuwenden, den die lettländischen Internisten Martin Sihle und Kristaps Rudzitis in der Medizin und medizinischen Philosophie Lettlands einnehmen.

Wir betonen oft, daß unserem Leben eine besondere Unrast, fiulle von Eindrücken und Geschehnissen eigen ist, ein Chaos, das eine Quelle dieses Stresses ist.

Meiner Ansicht nach lebten die Menschen seit jeher unter beständigem Stress: Kreuzzüge, Inquisition, Pestepidemien konnten kein "Goldenes 


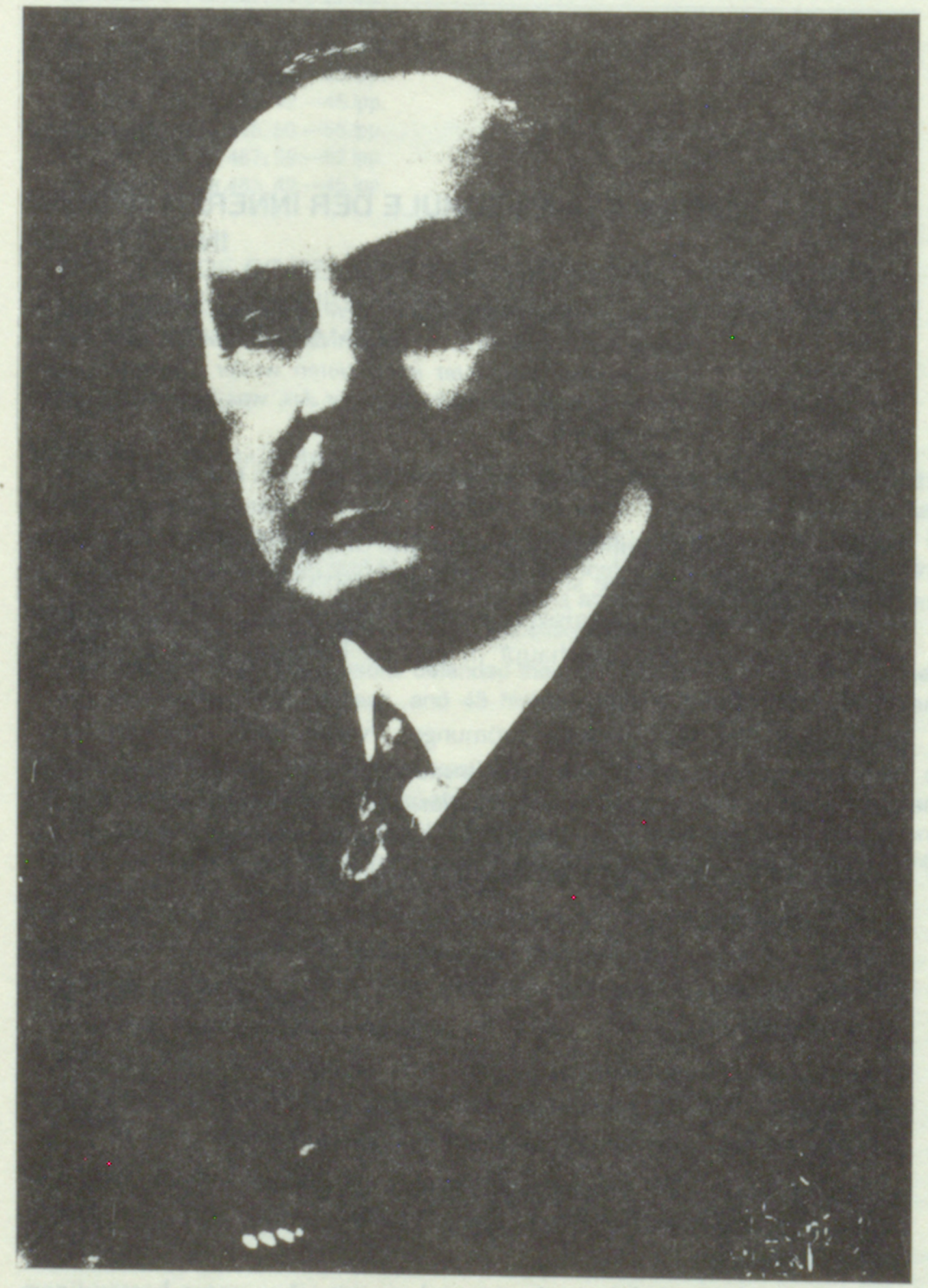

Martin Sihle 
Zeitalter" erzeugen. Wir schreiben, unser Zeitalter wäre ein Zeitalter der Widersprüche: neben der Wissenschaft existiere Irreführung.

Manchmal scheint es sogar, die Wissenschaft in der Medizin wäre am Versiegen. Die gesamte Geschichte der Wissenschaft - auch in Lettland ist ein Beispiel derartiger Widersprüche, des Entwicklungsganges und seiner Wege. Dies ist eine normale, sogar unumgänglich notwendige Erscheinung, die Vorbedingung eines Fortschrittes.

Materialismus, Metaphysik, Idealismus, begrenzter Praktizismus, ausgedehnte Philosophie, Scharlatanerie und das Streben nach der absoluten Wahrheit, all das gehört auch zum Gesamtbild unserer Medizingeschichte. Die Anfänge der inneren Medizin in Lettland beginnen in Rußland-den Petersburger und Moskauschen Schulen, auch den Europäischen Hochschulen, hauptsächlich in deutschsprachigen Ländern. Ihre Wurzeln sind auch in der Dorpater Universität vorhanden, wo unsere ersten Ärzte und Gelehrten ausgebildet wurden.

Man könnte anerkennen, daß Kristaps Helmanis, der bekannte Erforscher der Rotzkrankheit und der diagnostischen Eigenschaften des Maleins als Lettlands bedeutendster Medizin-Wissenschaftler zu bewerten ist. "Einen bedeutenderen Vertreter der medizinischen Wissenschaft hatten wir nicht", betont A. Viksna in seinem Vorwort zu dem Buch von Egons Dãrziñ š ${ }^{1}$. Diese Bezeichnung wählte A. Viksna daher, daß K. Helmanis vielleicht der einzige Medizin-Wissenschaftler Lettlands ist, dessen Name in der Geschichte der Medizin der ganzen Welt figuriert.

Wir besitzen viele andere Gelehrten, deren Name mit den Gesetzen der Chemie, Astronomie oder den Planeten eng verknüpft ist. In dieser Hinsicht ist K. Helmanis der einzige in der Medizin.

Die Kultur, Wissenschaft, auch Medizin, weist eine ganze Reihe vortrefflicher erfolgreicher Resultat und Wissenschafter auf, die bisher noch keine höchste Anerkennung gefunden haben, z.B. den Nobelpreis erhalten oder Mitglied der Academie Francaise geworden sind (z.B. sogar Victor Hugo war nie zu deren Mitglied ernannt).

Die Medizin Lettlands besitzt Persönlichkeiten, die bedeutende Werke und Ideen, besonders in ihrer Heimat und deren Geschichte, hinterlassen haben. Manche Gelehrten haben Beiträge zur Wissenschaft Europas und Amerikas geleistet: der Mikrobiologe Egons Dārziṇš hat Anerkennung in Amerika gefunden dank seiner Erforschung der Tuberkulose; Jēkabs Primanis hat mehrere Preise der Universität Pittsburg erhalten, darunter auch Anerkennungspreise der Studentenschaft. In Europa gehört zu solchen MedizinWissenschaftlern der Internist und Philosoph der Medizin Martin Sihle.

1 Dârziņ̌̌ E. Zemmers, Kalniņ̌̌, Helmanis. Dzive un darbi. - Riga: Zinătne, 1993. - 169 lpp. 
Geräume Zeit nach Absolvierung des medizinischen Instituts begannen mich die Werke von Märtinš Sihle zu fesseln, die damals entweder verboten oder der Vergessenheit anheim gegeben waren. Je mehr ich mich ins Lesen vertiefte, desto genauer ersah ich, daß viele philosophische Ideen meines Lehrers Prof. Kristaps Rudzitis, die uns so hinrissen, den Grundgedanken der Hauptwerke von Martin Sihle entstammen: "Über das Weltbild des Arztes" (Wien, 1934), "Das Urphänomen des Lebens" (Wien, 1935); "Was ist Seele?" (Wien, 1935), letzteres ist auch in lettischer Sprache erschienen. Die Forschungen der vergangenen Jahrhunderte erreichten ihren Höhepunkt gegen Ende des 19. Jh.: Man hatte eine "messbare, zählbare und begreifliche Welt entdeckt, wobei der kritisch-analytische Intellekt und der Rationalismus den Vorrang hatten". Mit diesen Worten machte M. Sihle der damaligen Medizin den Vorwurf, sich überaus enthusiastisch der Umfassung des Materiellen zugewendet zu haben. Die erzielten Erfolge der Medizin hat M. Sihle immer bewundert und praktisch ausgewertet.

Er bemerkte hierzu, diese Resultate riefen einen tiefen Spalt zwischen Arzt und Patienten hervor. In den heute technisch fortgeschrittenen Ländern, wo die Medizin vom wissenschaftlichen Standpunkt ein dem Verstand schwer begreifliches Niveau erreicht haben, ist der Spalt zwischen dem Seelenleben der Menschen und den "messbaren, zählbaren und begreiflichen" Vorgängen im menschlichen Organismus so unendlich tief geworden, daß dieses selbst M. Sihle verwundern würde.

Nach Sihles Überzeugung behindert eine derartig einseitig auf die Naturwissenschaft eingestellte Position die Gemütsbewegungen, das Denkvermögen und Begreifen des wahren Sinnes des Lebens. Das Streben M. Sihles ist auf ein solches Modell des Menschen gerichtet, das das Körperliche, Seelische und Geistige einschliessen soll. Sihle ist der Ansicht, ein jeder Student und Mediziner müsse sich an erster Stelle auf gründliche Kenntnis der modernen Medizinwissenschaft stützen, um in einer jeden Situation fähig zu sein, den Zustand des Kranken analysieren zu können, zu bewerten und die neuesten, entsprechenden Maßnamen in der Diagnostik und Behandlung zu wählen.

Nach M. Sihles Meinung ist die Zelle, die kleinste Einheit des Körpers, abhängig von drei Faktoren, die optimal koordiniert zu sein haben: ein bestimmtes Ausgangsmaterial, einschließlich Information und Nährprodukte; funktionsfähige Elemente und Kontroll-Faktoren, welche die Funktion der Zelle bestimmen.

Heutzutage könnten wir mit Leichtigkeit andere Definierungen anwenden: genetische Information, Außenweltumstände, Regulationsmechanismus, Enzyme, Hormone und reflexive Korrelation. Stimmt das Ausgangsma- 
terial nicht überein mit dem aktiven Material und der Kontrolle, ist der Mensch als krank anzusehen. Von diesem Standpunkt aus sind, nach der Meinung von M. Sihle, Synergismus und Antagonismus logisch unvereinbar, jedoch für den lebenden Körper eine notwendig miteinander verbundene Erscheinung.

Das Phänomen des menschlichen Lebens teilt M. Sihle in drei Schichten:

1. Das Reale=Oberflächenschicht.

2. Das Potentiale=Tiefenschicht.

3. Das Präpotentiale=der Urgrund=Ursache allen Geschehens, Anreger alles Geschehens².

Die reale Schicht ist sichtbar, anfühlbar. An dieser Schicht verfolgt der Arzt den Entwicklungsgang der Krankheit. Die potentiale Schicht besteht aus vielerlei einzelnen Potentialen, die sich in ihr auf verschiedene Art und Weise verankert haben und das Potential stimulieren. Je näher an der Oberfläche etwas liegt, desto stärker ist seine Einwirkung gefördert.

Die potentiale Schicht enthält außerdem potentiale Teile, die den ganzen Körper formieren. Sie umfaßt auch solche Werte wie ethische Prinzipien, Intellekt, Mangel an Gewissenhaftigkeit, Eigenliebe, Unsicherheit. Die Entwicklung dieser Eigenschaften hängt davon ab, wie tief sie in der potentialen Schicht liegen. Die Schicht der Potentiale ist auch das Arbeitsfeld des Arztes, da er an ihr alle Heilungsarten anwenden kann.

Vergleichen wir in Gedanken alles Gesagte mit der Wissenschaft der Gegenwart, wird es dem Leser leichtfallen, eine Analogie zur heutigen Konstruktion der Diagnostik, der klinischen Bewertung und Behandlung zu finden. M. Sihle befaßt sich außerdem auch mit Heilungs-Methaphysik.

Er behauptete, die Żelle vollführe schöpferische Arbeit und, daß ihr Potentialbereich reichhaltiger ist als derjenige der Realschicht, daß die Zelle Geist und Seele hat und zielbewußt auf gewisse Reizungen reagieren kann.

Es bleibt mir nur übrig, den Skeptikern, die über diese Idee lächeln, auf den Begründer derimodernen Sozialbiologie in Amerika, Richard Ardrey, hinzuweisen, der in'seinem Buch "Der territoriale Imperativ" nachweist, daß sogar solche Eigenschaften wie der Rationalismus vor allem in einzelligen Organismen bei ih́rem Teilungsprozeß beobachtet werden können. In der Darlegung seiner Ansichten über den Menschen als ein Ganzes bedient sich M. Sihle zweier Begriffe: Inspekt und Aspekt. Mit Inspekt bezeichnet er die analytischen Forschungsmethoden. Bei der Inspektion, oder dem

2 Sihle M. Öber das Weltbild des Arztes und den Sinn der Krankheit. - Wien; Leipzig; Bern; 1934. - S.109. 
Einblicken, versuchen wir das zu inspizierende Objekt nach Möglichkeit aus der nächsten Nähe zu besehen, um es in allen Details zu erblicken.

Aspizierend oder besichtigend entfernen wir uns, in Gegenteil, von ihm, um es besser in größeren Dimensionen genauer zu erblicken und sein Gesamtbild zu erfassen ("singulare sentitur, universale intellegitur"). Inspekt und Aspekt waren beliebte Begriffe auch von Prof. K. Rudzitis, mit denen er oft seine Vorlesungen an Studenten des IV. Lehrjahres im Herbstsemester begann. In der Interpretation des Professors bedeutete das eine sorgfältige, physikalische und laboratorische Untersuchung des Kranken, also den Inspekt. Der Aspekt bedeutete hingegen alle bereits vorhandenen Analysen, eine genaue Anamnese und die Bewertung der Person des Kranken.

M. Sihle betonte die Wichtigkeit der korrelativen Pathologie. Das heißt, man könne keinen Prozess gesondert beleuchten, sondern nur zugleich in deren gegenseitiger Abhängligkeit. Daher muß man sich auch bei der Behandlung auf die Korrelationspathologie stützen, auf die Bewegung der Körpersäfte, und dazu gibt es drei Wege: 1. Kapillaren; 2. Flüssigkeiten der Gewebe; 3. die Zelle. Nur eine gleichzeitige Anwendung von Methoden, die aktive Hyperämie und aktive Hyperlymphie hervorrufen können, erlaubt uns, einen medizinischen Heilungseffekt zu erzielen. Nach diesen Anschauungen beeinflussen Hyperämie und Hyperlymphie die amorphen Kolloide der Gewebe, von denen die optimale Funktion der Organe abhängt der Prozess des Anschwellens und der Verflachung der Kolloide muß man mit dem übereinstimmenden inneren Zustand des Organismus und seinen Belastungen im gegebenen Moment rechnen. Kommen bei diesem System Abweichungen vor, Prozesse wie z.B. plötzliche Kristallisation, können ernstliche Folgen eintreten.

Seine Ideen über die obengenannten drei Wege von der Zirkulation der Kolloiden und der Gewebe wurden Prof. M. Sihle grundlegend bei seinen Forschungen über chemische Kolloide bei Prof. Schade in Kiel. Sie endeten mit der Dr.med. Dissertation "Klinische und kolloidochemische Forschungen über paradoxale Reaktionen", die K. Rudzitis, damals Assistent an der Klinik für innere Krankheiten, im Jahre 1932 in Riga verteidigte. Nach M. Sihles Ansicht waren die Korrelationen auch von Bedeutung für die Hygiogenese, welche M. Sihle höher schätzte als die Sanogenese. Unter der Hygiogenese verstand er die Entwicklung und Kräftigung der Gesundheit, stellte sie der Sanogenese gegenüber, d.h. dem Gesundheitsprozeß. Nimmt man das Gesagte in Betracht, wird es klar, weshalb Prof. M. Sihle in der praktischen Medizin so großen Nachdruck auf zwei Sachen legte: Bewertung des Menschen als ein Ganzes und physikale Therapie, zur Wirkung auf die Zellen und die Gewebe. Von großem Wert hielt er Balneotherapie, Massage, 
mancherlei Umschläge, Salz- und Moorbäder, sowie Abreibungen der Haut mit einer scharfen Bürste. Noch heute kennt man die "M. Sihle Bürste."

In seinen Überlegungen über die Synthese der Medizin und synthetisches Denken stützt sich M. Sihle auf die Werke zahlreicher Philosophen und Schriftsteller der Antike und folgert aus ihnen seine Ideen. Einer von diesen war der griechische Poet und Philosoph Xenophon von Kolophan (6.-5. Jh. a. Chr. G.; nicht verwechseln mit dem griechischen Schriftsteller Xenophon aus Attika; ungefähr 430-355; noch mit Xenophon von Ephesus cirka 2. Jh. a. Chr. G., der mit seinem Liebesroman "Ephesiaka" Anerkennung erworben hat). Xenophon von Kolophan betonte nur der Verstand wäre fähig das Unsichtbare sichtbar zu machen.

Die liebsten Philosophen der Neuzeit waren für M. Sihle Schopenhauer, Fichte, Aristoteles und Hegel, Nietzsche und Schelling, sowie einige anderen. I. Kant nimmt bei M. Sihle einen besonderen Platz ein. Während er die erwähnten und noch andere, oft zitiert und von ihnen spricht, diskutiert er mit Kant, erhebt Einspruch gegen dessen Apriorismus den er als Brücke ansieht, die vom Empirismus zum Idealismus führt und als nicht endgültig durchdachte Philosophie gelten kann. Kant anerkennt "a priori" Existenz, glaubt jedoch, die Existenz Goltes wäre unbeweisbar. M. Sihles Ansicht nach bedeutet "beweisen" nicht einen gewöhnlichen Versuch etwas zu beweisen, sondern etwas mit Hilfe physiko-mathematischen Methoden nachzuweisen, etwas messend, abwiegend oder befühlend, jedoch dabei vergessend, daß wir in unseren Messungen, unserem Abwiegen und Befühlen selbst unvollkommen sind. Nach M. Sihles Ansichten, erübrigt es sich beständig daran zu erinnern, daß die Existenz Gottes unbeweisbar ist, - sie bedarf überhaupt nicht bewiesen zu werden, ist als "stetige Gegenwart" aufzufassen. Nach M. Sihles Meinung findet am Krankenbett stets ein dreifaches Konsilium statt, an dem der Patient, der Arzt und Gott teilnehmen (das entspricht dem "a priori" von Kant).

Vom Standpunkt des Arztes betrachtet M. Sihle auch das Gebet. Es wäre ein Mysterium, daher müsse man dabei allein sein und sich in sich selbst zu vertiefen. Beim Beten ist das "eigene Ich" auf der Suche unter engeren Beziehung zu seinem Urquell, den man Gott nennen kann.M. Sihle übt scharfe Kritik an zwei solchen Eigenschaften wie Intoleranz und Ignoranz. Ignoranz sei ein Zeichen von Unwissenheit, doch in der Medizin ist Wissen die Hauptsache, verstehen und nach Wissen streben. Mit diesen Gedanken beendet M. Sihle seine Überlegungen zum Charakterbildnis des Arztes (Weltbild) und betont, daß wir den Begriff "ignorabimus" viel zu häufig anwenden, es müsste durch den Begriff "gnorabimus" ersetzt werden. Das bedeutet, mit anderen Worten, die Ignoranz und der Obskuran- 
tismus müssen vom Wissen abgelöst werden. Wie bereits gesagt, sind die angeführten, und noch andere, Ideen M. Sihles in den Werken von Prof. K. Rudzitis übernommen und fortgesetzt worden.

Bei eingehender Durchsicht der klinischen Arbeiten von Prof. K. Rudzitis, beginnend mit seinen Forschungen über paradoxale Reaktionen, Silizium, Bindegewebe, Aterosklerose, Holesterin, fokale Infektion und zum Schluss seiner letzten wissenschaftlichen Monographie "Heparinozyten" (Riga, 1959) wird der synthetische Zugang des Professors zu Prozessen und Menschen deutlich. Es ist als ob Prof. K. Rudzitis die Ideen und philosophischen Betrachtungen M. Sihles der praktischen Heilkunde näher gebracht habe.

Als Arzt ermunterte K. Rudzitis zu einem einfachen Zugang zum Patienten die Aufmerksamkeit leicht zu konstatierenden Symptomen zu widmen, nicht damit zu beginnen sich in vielerlei komplizierte Analysen zu vertiefen. Bei seinen Vorlesungen und Visiten demonstrierte er meisterhaft so bekannte Symptome wie Magenblähung, Geräusche in den Gelenken bei Kopfbewegungen, vereinzelte Ausschläge auf dem Gesicht, die mit der Krankheit selbst und ihrem Verlauf verbunden sein können. Dabei formulierte Prof. Rudzitis nicht nur die Diagnose sondern auch díe Pathologie, wobei die fokale Infektion stets eine besondere Rolle spielte. Ein derartiger Zugang erlaube Prof. K. Rudzitis manchmal eine unerwartete Wendung dort festzustellen, wo Kollegen und Konsultanten sich zu sehr in genaue Analysen vertieft und dabei in den Labyrinten der gewonnenen Daten verirt hatten.

Eine plötzliche, unerwartete Änderung der Diagnose führte manchmal zu therapeutischen Konsequenzen, so z.B. in schwierigen Fällen des Einführens der Magensonde um den Magen von übermässiger Luft zu leren falls der Kranke an Aerophagie litt. Dies besserte die Atmung, Blutzirkulation und das Allgemeinbefinden des Patienten.

Der Professor hatte von M. Sihle die Anwendung der physikalischen Therapie übernommen und sie vervollkommnet. Er betonte dabei besonders die üblichen Arzneimittel-Anwendung, z.B. das Duschen, mit gutem Erfolg in der Praxis Aeroplagie, half Meteorismus und verschiedene Magenschmerzen zu verhindern. Prof. K. Rudzitis fand es nicht so wichtig einem Kranken, der viel Schleim absonderte, dabei ein Hilfsmittel zu verordnen, sondern ihn in der richtigen Lage zu betten. Lesen wir in den Lehrbüchern und Abhandlungen für das Jahr 1993 nach, werden wir bemerken, daß bei Behandlung von Bronchektase, chronischer Bronchitis oder Lungenabszess an erster Stelle eine richtige Drainage vorzunehmen ist und dem Kranken im Bett die nötige Lage dazu zu verschaffen.

Die Ansicht von M. Sihle über den synthetischen Zugang zum Kranken hat K. Rudzitis in das geflügelte Wort transformiert der Kranke müsse nicht 
anders untersucht werden als "a capita ad calcem", d. h. von Kopf bis Fuß und nicht, wie der Professor hinzufügt "a clavicula ad umbinem", d. h. vom Schlüsselbein bis zum Nabel. In seinem Buch "Heparinozyten" hat K. Rudzittis ein glänzendes Beispiel synthetischen Denkens angeführt.

Beschrieben und nachgewiesen sind in diesem Werk die engen funktionalen Verbindungen zwischen den Blutformelementen die solche Begriffe wie Assoziationen bilden: eine Assoziation der Neutrofile, Lymphozyten und Eosinofile und noch andere.

Hierzu ist zu sagen, dass sich die Erforschungen bösartiger Neubildungen von einer Schülerin Prof. Rudzitis, Vera Rudzite (keine Verwandschaft!) gerade aufgrund der obengenannten Forschungen vollzogen, wobei bewiesen wurde, dass Triptofan bedingte bösartige Blutzellen in Korrelation mit bösartigen Zellen von gewissem Einfluß auf den Stoffwechsel des Triptofans sind. Besonders war es das Studium des Metabolismus des Kinurinins, das Prof. V. Rudzite in Europa zu einer anerkannten Wissenschaftlerin gemacht hat.

Dogmen hat Prof. K. Rudzitis immer angezweifelt. In der Zeit, als die Holesterintheorie dominierend gewesen war, betonte der Professor, er müsse bei Holesterin-Verbrauch Gleichgewicht herrschen zwischen dem exogenen und endogenen Holesterin. Eine völlig holesterinlose Diät sei nicht ratsam.

Auch in seinem moralischen und ethischen Verhalten war der Professor ein Nachfolger von M. Sihle. Konkret und sachlich vermied er hochtrabende Phrasen und leere Worte, war immer bereit einem jeden, auch bei Kleinigkeiten, zu helfen. Er lehrte, sich nicht mit Bagatellen abzugeben, die von anderen entschieden sein können, sich nicht in Sachen zu mischen, die nicht zu den Aufgaben eines Leiters gehören und fügte oft schmunzelnd hinzu die Hauptaufgabe des Leiters wäre nicht zu leiten sondern, Tag für Tag, dafür zu sorgen, daß die Arbeit störungslos verläuft. Wie treffend stimmt das überein mit einem Ausspruch des Lao Tse ein guter Herrscher wäre derjenige, dessen Abwesenheit seine Untertanen nicht bemerken, ein schlechter Herrscher wäre der, in dessen Abwesenheit Verwirrung entsteht.

In seinem Verhalten zu seinen Schülern, Kollegen und zum Leben selbst, dominierten bei Prof. K. Rudzitis Toleranz und Wissen. Er verstand es zu einem den Zugang zu finden, oft genug Menschen von verschiedenem Charakter, Temperament und Interessen zu vereinen. M. Sihle, K. Rudzitis und die Kultur.

In dieser Hinsicht können wir auch interessante Parallelen finden. M. Sihle war ein ausnehmend gebildeter Mensch. Das ist auch in dem Buch von Th. Brugsch "Arzt seit fünf Jahrzehnten" (Berlin, 1957) besonders betont. M. Sihle beherrschte die klassischen Sprachen - Latein und Griechisch. 
Seine erste Sprache war Deutsch, auch sprach er frei Russisch und, aus Odessa nach Lettland zurückgekehrt, vervollkommnete er das Lettisch.

Die bevorzugte von den Künsten, die ihn bezeichnete, war, zweifellos, die Musik. Er spielte selbst oftmals Klavier, traf sich mit Musikern. Die Enkelin von M. Sihle, Irina Küssman-Sihle, die mehrmals Riga besucht hatte, erzählte, daß in der Wohnung des Professors im Oberstock eines Hauses in der Elizabeth-Straße (an diesem Hause ist jetzt eine Denktafel angebracht) habe in einem der Zimmer ein Konzertflügel gestanden, auf dem Prof. Sihle und seine Gäste oft gespielt hätten. An den häufigen Musikabenden spielten und sangen bekannte Musiker, Geiger und Sänger, wie z.B. Pauls Sakss. Im Leben Prof. M. Sihles war die Musik das beste Mittel zur geistigen Entspannung. Der selige Dozent V. Bümeisters hat seinerzeit erzählt, er entsinne sich der Weihnachtstage in der Klinik für innere Krankheiten. An jedem 25sten Dezember erschien am Morgen ihr Leiter Prof. Sihle. Im Korridor stand bereits ein rechtzeitig hergebrachtes kleineres Instrument, meist eine kleine Orgel, auf dem, ohne etwas zu sagen, Prof. Sihle einen Weinachtschoral vortrug. Die Kranken und das um diese Zeit anwesende Personal hörten mit verhaltenem Atem zu.

Auch im Leben Prof. K. Rudzitis hatte Musik große Bedeutung, er hatte auch eigene Kompositionen verfaBt z.B. Variationen über lettische Volkslieder. Dennoch standen für ihn die Lexikographie, respektive die medizinische Terminologie an erster Stelle.

Seine Arbeit auf diesem Gebiet beschränkte sich nicht auf die Medizin und erstreckte sich auf die Kultur des Volkes. In seinen philosophischen Schriften behandelte er Geschichte, Migration der Völker, Herkunft der Balten, das Gemeinsame der lettischen Sprache mit dem Sanskrit und dem Griechischen und noch andere originelle und scharfsinnige Hypothesen. Obwohl viele dieser Hypothesen nicht bewiesen wurden, regen sie trotz dem an nachzudenken und zu überlegen.

Prof. K. Rudzitis Werk "Terminologia medica" (über 53000 termini technici) ist ein Unikat, das einige dieser Art in der damaligen Sowjet-Union und eine der Raritäten in Europa. Es ist einmalig dank dem dreisprachigen Text-Latein, Lettisch, Russisch - eine wahre Schatzkammer an Bezeichnungen, die nicht nur Medizin betreffen, sondern auch viele der Medizin nahe Gebiete umfassen, wie z.B. Linguistik, Chemie, Biologie, Physik, Astronomie und andere.

Prof. K. Rudzitis konnte einen ganzen Tag, sogar tagelang, über eine einzige Bezeichnung nachdenken, über eine einzige Satzstellung um sie möglichst präzise zu formulieren, linguistisch zu vervollkommnen um den Inhalt erfassen zu können. 
Prof. K. Rudzitis synthetischer Zugang zur Medizin und der gesamten Kultur erlaubte es ihm bei seinen wissenschaftlichen Forschungen mit Physikern, Chemikern, speziell jedoch mit Astronomen nahe Bande zu knüpfen. Im Jahre 1965 fand in Riga, unter der Leitung von Prof. K. Rudzitis, das I astrobiologische Symposium statt, auf dem zum ersten Mal die Rede war von dem solaren Einfluß auf die Prozesse in einem lebenden Organisrous. K. Rudzitis schätzte die Ideen von A. Čiževski sehr hoch.

Hier ist zu sagen, daß für A. Čiževskis Versuche die Revolutionen in aller Welt von kosmischen Vorgängen beeinflußt zu sein, ihm zum Lohn, langjärige Verbannung bestimmt wurde.

K. Rudzitis pflegte nicht nur von solaren sondern auch von telluren und selenen Einflüssen und der Wirkung kosmischer Strahlen auf den Menschen zu sprechen.

Prof. K. Rudzitis reiches enzyklopädisches Wissen und von M. Sihle übernommenen phylosophischen Ansichten widerspiegeln sich in seinen Lehrbüchern der rationellen Medizin, deren glänzendes Beispiel sein Buch "Grundlagen der Diagnostik und Preambel der Therapie." (Riga, 1972), das besonders in der zweiten Hälfte die den Symptomen, Diagnosen und Fehldiagnosen und deren Gründen sowie dem Nachdenken über den gesunden und kranken Menschen gewidmet ist. Darin teilt Prof. K. Rudzitis die Anschauungen des Hippokrates über den inneren und äußeren Arzt. Sie erinnern an M. Sihles Pathologie der Korrelationen, die Zusammenhänge der Konstitution mit den Krankheiten und spricht außerdem über die äußeren Einflüsse - der Umwelt, Sonne, Wasser, Luft, Klima und meteorologischen Faktoren.

Eigenartig sind auch die Ansichten von Prof. K. Rudzitis über Nahrung, Regime, medikamentöse Therapie und noch andere Faktoren. Liest man heutzutage in diesem Teil des Buches nach, kann es nur wundern wie modern, zeitgemäß und den gegenwärtigen Ansprüchen der Wissenschaft dieselben entsprechen. Hier wäre wieder auf M. Sihle zurückzukommen und auf die Bande seiner Ansichten mit unserer Zeit.

In diesem Zusammenhang will ich auf das Buch von W.Spradlin und P.B.Porterfield "The search for certainty" (Springer-Vlg., 1984) hinweisen. Beide Autoren sind große Wissenschaftler, der erste Physiker, der zweite Biologe, die in Kürze den Entwicklungsgang der gesamten Wissenschaft darlegen.

In der frühesten Welt der antromorphischen Wörter dienten diese zu Instrumenten, welche die Menschen von der Umwelt absonderten und sie in verschiedene Begriffe und Dinge teilten. Es entstand ein System von Anschauungen das sich an die Idee stützte, das Leben des Menschen wer- 
de von andern, außerhalb unserer Existenz befindlichen, Kräften gelenkt und bestimmt. Kopernikus, die Gelehrten der Renaissannce, Darwin, Freud und noch andere, schufen Theorien, verfertigten Instrumente, es begann das Entstehen der sogenannten modernen Wissenschaft, bei der wir die Information auf dem Wege von Messungen erhalten. Zu Beginn unseres Jahrhunderts kam zur Wissenschaft die Relativitäts-Theorie von Einstein hinzu. M. Sihle bezweifelte bereits in den dreißiger Jahren den besonderen Nachdruck auf präzise Messungen. Auch die Verfasser des 1984 erschienenen obengenannten Buches teilen die Ansicht, daß der auf Messungen gelegte übermässige Nachdruck für die Welt der mechanischen Formen und Funktionen typisch ist und zu der paradoxalen Situation führt in der alle Messungen relativ werden.

Bei einem solchen relativistischen Zugang beginnen wir selbst mit unserer Welt, Form und Funktion zu verschmelzen. Das Sein oder Nicht-Sein, das eigene SELBST oder kein SELBST, Bewegung und Ruhezustand werden zu verschwommenen Begriffen deren Auslegung stets zusätzliche Erklärung verlangt: in Beziehung auf das "WOGEGEN"? Der moderne theoretische Rahmen vereinigt alle lebenden und anorganischen Systeme zu einer Ununterbrochenheit und verkleinert hiermit die Rolle des Menschen als ein absoluter spezifischer lebender Organismus oder schließt sie vollständig aus.

Jedes Absolut oder Ursache und Effekt oder Resultat, wird zu Illusionen und kann nur bewiesen werden durch retrospektives Anordnen der Vorgänge.

Die neue konzeptuale Umrahmung des 20. Jh. gestattete die Vereinigung der Flächen des Mikrokosmos und des Makrokosmos, den Makrokosmos der interstellaren Bande und unsere Vorrichtungen und rezeptiven Organe in eine gemeinsame integrale Strömung zu bringen.Wobei wir nicht mehr unbeteiligte oberflächliche Beobachter bleiben, sondern zu Teilnehmern der Vorgänge werden. Das Protoplasme unserer Zellen, das fähig ist z.B. Information durch ein Elektronmikroskop oder Radarmikroskop aufzunehmen ist keine Perzeption und Erkenntnis (wie es in der alten Welt der relativen Messungen war), sondern eine kurze Harmonie der gegenseitigen Beeinflußung der Kraftflächen.

Beim Lesen dieser Worte stellt man sich unwillkürlich die Frage, ob sie nicht M. Sihle geschrieben hat und sie nur in ihrer Form un bedeutend modernisiert wurden.

Auch die Ansichten von W.Spradlin und P.B.Porterfield über den Tod sind eng verknüpft mit M. Sihles Gedanken. Vielen von uns gilt der Tod als absolutes Ende, als der letzte Schlag für unsere Gedankenwelt. Anderen eröffnet der Tod eine neue Umrahmung in der wir uns der Harmonie der Fluide aller Lebewesen anschließen und bei diesem Prozeß scheinbar ewig werden. 
Diese Konzeption überwindet den Spalt zwischen Wissenschaft und Religion, katalisiert andere Einflüsse, diejenigen, die wir Kunst, Wissenschaft und Philosophie nennen.

In der Musik bedeutet der Kontrapunkt eine gleichzeitige Bewegung mehrerer selbständiger Stimmen, die ein aufeinander abgestimmtes harmonisches Ganzes bilden.

Als charakteristische Form des Kontrapunktes bezeichnet man die Fuge. M. Sihle wählte für eine seiner späteren Arbeiten, die er erst in den Kriegsjahren vollendet hatte im Titel das Wort "Kontrapunkt" - "Der Kontrapunkt des Lebens", zur Devise dieses Buches die Worte Otto von Bismarks: "Ich muß wahr sein um Mut zu haben." In diesem Buch ist M. Sihle bestrebt seinen vorherigen Werken das Fazit zu ziehen oder ihre Zusammenfassung zu unternehmen.

Bereits im Vorwort ist es vermerkt, daß ein synthetischer Zugang zur Erforschung des Lebens eine hinreißende Betätigung ist, die sich nicht nur auf den Arzt bezieht, sondern auf einen jeden der dieses Problem antastet, sei er Biologe, Philosoph, Historiker oder jemand anders. An vielen Stellen diskutiert M. Sihle mit dem Positivismus, der verkündet, daß in der Forschung keine "Tiefe" bestehe, daß alles nur "Oberfläche" sei und es daher nur möglich wäre das "Bestehende" und das "Gegebene" zu erforschen. Jedoch nicht dasjenige, das sich darunter verbirgt.

Diese Thesis der Positivisten betrachtet M. Sihle als direkten Gegensatz einer synthetischen Auffassung des Lebens und betont dabei, in diesem Buche mache er den Versuch die Gesetzmässigkeit der Vorgänge im Leben aus der Perspektive der Kunst, besonders der Musik, zu betrachten. Das ist ein interessanter und neuer Wendepunkt in der Philosophie von M. Sihle die wohl seine Überlegungen über Leben und Kultur und die Mission des Arztes beschließt.

In der Musik ist, ebenso wie in der bildenden Kunst, die Auffassung der Perspektive, die für den Maler so wichtig ist, aus der die Vorstellungen über Proportionen entstehen, doch diese kann man nur dadurch gewinnen, daß man Kunstwerke aus einiger Entfernung betrachtet oder sich ein Musikwerk von Anfang bis zu Ende anhört. Unablässig kommt M. Sihle auf die Probleme von Krankheit und Gesundheit, von Leben und Tod; Geist und Seele zu sprechen betrachtet sie dabei in neuen, einzelnen Dimensionen und bleibt dennoch seinem synthetischen Zugang zu all diesen treu...

Anfang und Ende des Lebens von M. Sihle sind eigenartig, verbunden mit dem Nirgendland. Sein Großvater war ein Findling gewesen, dem ein 
Knecht auf dem Gute den Namen gab und dabei sagte: "Du bist rund wie eine Eichel also wirst du Eichel heißen" (ein Wortspiel: im Lettischen heißt die Eichel "Zile"). Am 12. Mai 1945 verschied Prof. M.Sihle in Deutschland in der Stadt Unsleben und wurde auch dort bestattet. Ganz sonderbarer Weise war seine Grabstätte bald darauf vernichtet worden und ist unbekannt geblieben.

Ebenfalls im großen Nirgendland verschollen. Im Jahre 1945, dem Todes-jahr von M. Sihle, wurde in einem ganz anderen Erdteil, Amerika, in New York, Duglas R.Hofstadter geboren. Er studierte die französische Sprache, Physik, Mathematik, Kunst, Musik und lernte in der Wissenschaft den synthetischen Zugang zu allen Geschehnissen kennen. Die liebsten Komponisten von D.R.Hofstadter waren Chopin und Bach. Er spielte selbst gut mehrere Musikinstrumente, meist Klavier, erzielte merklichen Erfolg mit vielen mathematischen Abstraktionen und in der theoretischen Physik. Ein Beispiel seines synthetischen Denkens ist das 1979 erstmalig publizierte und mehrmals in weiteren Auflagen erschienene Buch von D.Hofstadter: "Gödel, Escher, Bach."

Schon der Titel des Buches weist darauf hin, daß der Autor in philosophischen Diskussionen die unlösbaren Bande darlegt, welche die pure Wissenschaft - die Mathematik, die Gödel vertritt (Kurt Gödel; 1906-1978, deutscher Mathematiker), die Malerei, die Escher vertritt (Mauritius Cornelis Escher; 1898-1972, holländischer Graphiker) und die Musik (Jochan Sebastian Bach 1685-1750), verknüpfen.

M. Sihle hat, außerhalb Lettlands, in der Kriegszeit seine letzten Über-legungen zu schreiben begonnen doch nicht beendet: "Das Schicksal der ständigen Lebensdauer des Menschen und seine Berufung", mit dem Subtitel "Schlußfolgerungen eines Forschers der Synthese des Lebens gesehen vom Standpunkt der eigentlichen Tätigkeit des Arztes." M. Sihle schreibt, hat er sich gegen Ende seines Lebens abermals dem "Problem der Probleme" zugewandt, das soll bedeuten, daß es für einen Arzt nicht am Allerwichtigsten ist wer oder wie der Mensch ist, sondern das, was er als Mensch bedeutet.

Diese Worte sind noch heutzutage aktuell. Sie stimmen überein nicht nur mit der Wissenschaft, egal ob zu ihr ein analytischer oder synthetischer Zugang besteht, sondern beziehen sich auf das Fundament unserer Moral, Ethik, Kultur und Existenz.

Erneut begegnen wir diesen Fragen in der Zeit unseres dritten Erwachens. Darüber schreibt Jānis Stradiṇ̌̌ in seinem Buch "Das dritte Erwachen" (Riga, 1992): "Ein Erwachen ist stets schön und romantisch, es bleibt für immer in Erinnerung, als eine Krönung unseres Lebens, als etwas Erhabenes, Unerreichbares und - leider - Einmaliges. Die Zeit der Arbeit ist prosaisch, trübe, monoton und schwierig, bringt nicht allemal einem jeden 
Genugtuung. Und trotzdem, trotzdem: das Erwachen ist zu Ende, die tägliche Arbeit muß getan werden und man muß das tragen, was sich das Volk erwählt hat - das Kreuz der Freiheit."

\section{LITERATUR}

Brugsch K. Arzt seit fünt Jahrzehnten. - Berlin, 1959.

Dărziṇš E. Zemmers, Kalniṇš, Helmanis: Dzîve un darbi. - Riga: Zinâtne, 1993.

Gezer A.L. Martin Sihle: Versuch einer Bioergographie (Dissertation). - Münster, 1981.

Hofstadter D.R. Gơdel, Escher, Bach: an Eternal Golden Braid. - Great Britain: Penguin Books, 1979.

Lazovskis I. lekškigigo slimỉbu pamati. - Riga: Zvaigzne, 1985.

Lazovskis I. Profesors Kristaps Rudzitis // Veseliba. - 1989, - Nr. 4. - 14.-15. Ipp.

Rudzitis K. Diagnostikas pamati un terapijas preambula. - Riga: Zvaigzne, 1972.

Рудзит К. Гепариноциты в клинике и эксперименте. - Рига, 1959.

Rudzitis K. Kliniski un koloidkimiski pētijumi par paradoksăm reakcijăm. - Riga, 1932.

Rudzitis K. Terminologia medica: 1-2. - Riga, 1973; 1977.

Sihle M. Das Urphănomen des Lebens. - Wien, 1933.

Sihle M. Der Kontrapunkt des Lebens. - Deutschland; Polen, 1941-1943.

Sihle M. Schicksal und Sendung des Unvergănglichen im Menschen. - Deutschland; Polen, 1941-1943.

Sihle M. Über das Weltbild des Arztes und den Sinn der Krankheit: Ein Appell zur Lebenssynthese. - Wien, 1934.

Spradlin W.W., Porterfield P.B. The Search for Certainty. - Springer Verlag, 1984.

Stradinš J. Trešã atmoda: Raksti un runas 1988.-1990.gadă Latvijă un par Latviju. - Rĩga: Zinătne, 1992. - 405 lpp.

Чижевский А. Земное эхо сопнечных бурь. - Москва, 1973.

Prof. Dr.habil.med. IImārs LAZOVSKIS

Martas iela 9-7

Riga, LV1011

Latvija 
